\title{
Lesiones precursoras de malignidad gástrica y su asociación con hábitos alimentarios
}

\section{Precursor Lesions of Malignant Gastric Cancer and Associations with Eating Habits}

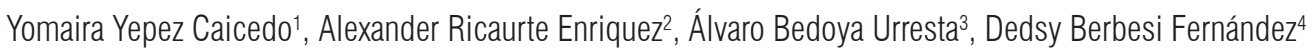

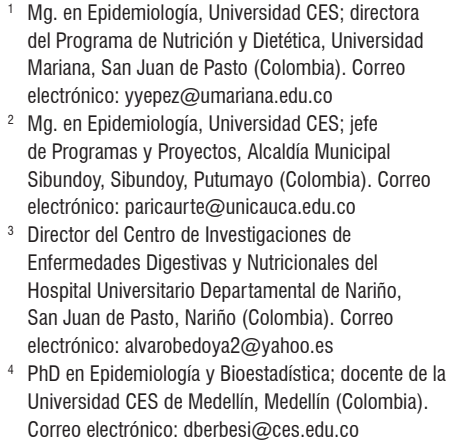
del Programa de Nutrición y Dietética, Universidad Mariana, San Juan de Pasto (Colombia). Correo electrónico: yyepez@umariana.edu.co

2 Mg. en Epidemiología, Universidad CES; jefe de Programas y Proyectos, Alcaldía Municipal Sibundoy, Sibundoy, Putumayo (Colombia). Correo electrónico: paricaurte@unicauca.edu.co

${ }^{3}$ Director del Centro de Investigaciones de Enfermedades Digestivas y Nutricionales del Hospital Universitario Departamental de Nariño, San Juan de Pasto, Nariño (Colombia). Correo electrónico: alvarobedoya2@yahoo.es

4 PhD en Epidemiología y Bioestadística; docente de la Universidad CES de Medellín, Medellín (Colombia). Correo electrónico: dberbesi@ces.edu.co

\begin{abstract}
Resumen
Introducción: se determinó la asociación entre los hábitos alimentarios y la presencia de lesiones precursoras de malignidad gástrica (gastritis crónica atrófica, metaplasia intestinal y displasia leve), en hombres y mujeres entre los 30 y los 60 años de edad que acudieron a consulta de gastroenterología en el Centro de Investigación de Enfermedades Digestivas de la ciudad de Pasto (Nariño) durante el último trimestre del año 2015 y el primer semestre del año 2016. Metodología: estudio analítico transversal en el que se analizaron variables histológicas, sociales, demográficas, antropométricas y alimentarias. Se realizó un análisis descriptivo, bivariado y multivariado por medio de la odds ratio (oportunidad relativa) cruda y ajustada con un IC del $95 \%$. Resultados: el 35,5\% de los 231 pacientes encuestados fueron diagnosticados histológicamente con dichas lesiones; la edad promedio no superó los 46 años; predominó el sexo femenino con $57,1 \%$; el $32,5 \%$ de los pacientes tenía una formación de nivel profesional y el 58\% estaba afiliado al régimen subsidiado. Estas lesiones se asociaron con el consumo de cereales y grasas por encima de los 10 y 8,5 intercambios (OR 2,20; IC del 95\%: 1,08-4,47 y OR 2,52; IC del 95\%: 1,28-4,94), respectivamente. La incorporación de sal a las comidas servidas aumenta la probabilidad de presentar lesiones estomacales en 1,94 (IC del 95\%: 1,03-3,66). El consumo regular de vegetales por encima de los 2 intercambios reduce la probabilidad de presentar lesiones estomacales (OR 0,53; IC del 95\%: $0,27-0,99)$. Conclusión: con la investigación, se pretende aportar conocimientos acerca de diferentes factores que pueden participar en la iniciación, promoción y progresión del cáncer gástrico.
\end{abstract}

\section{Palabras claves}

Lesiones precursoras de malignidad, hábitos alimentarios, cáncer gástrico.

\begin{abstract}
Introduction: Associations between dietary habits and precursor lesions of gastric malignancies including chronic atrophic gastritis, intestinal metaplasia and mild dysplasia were identified in men and women between the ages of 30 and 60 who came to the gastroenterology department of the Center for Research on Digestive Diseases of the city of Pasto, Nariño in the last quarter of 2015 and the first half of 2016. Methodology: This is a cross-sectional analytical study in which histological, social, demographic, anthropometric and dietary variables were analyzed. Descriptive, bivariate and multivariate analyzes were performed using crude and adjusted odds ratios with a $95 \% \mathrm{Cl}$. Results: Of the 231 patients surveyed, $35.5 \%$ were diagnosed histologically with precursor lesions. The average age was 46 years old, and $57.1 \%$ of the cases were women, $32.5 \%$ were professionals, and $58 \%$ were affiliated with the subsidized health care scheme. These lesions were associated with cereal consumption of above 10 exchanges (OR 2.20, 95\% Cl: 1.08 - 4.47) and with and fat consumption above 8.5 exchanges (OR 2.52, 95\% Cl: 1.28-4.94). Adding salt to meals increased the likelihood of developing stomach lesions by 1.94 (95\% Cl: 1.03-3.66). Regular consumption of vegetables for more than two exchanges reduced the likelihood of stomach lesions (OR 0.53; 95\% Cl: 0.27-0.99). Conclusion: This study aims at contributing knowledge about factors that involved in initiation, promotion and progression of gastric cancer.
\end{abstract}

\section{Keywords}

Precursor lesions of malignant cancer, eating habits, gastric cancer. 


\section{INTRODUCCIÓN}

El cáncer gástrico se considera una prioridad en salud pública, al ocupar el quinto lugar en incidencia (después del cáncer de pulmón, mama, colorrectal y próstata) y el tercero en mortalidad (después del cáncer de pulmón e hígado). Más del $73 \%$ de los casos de cáncer gástrico se producen en Asia; Europa aporta casi el 15\% de la carga mundial, mientras que América Central y del Sur contribuyen con un 7\% (1). En el departamento de Nariño, especialmente en la zona andina, donde se encuentra ubicada el $81,7 \%$ de la población, se ha reportado una incidencia de cáncer gástrico de 150 casos por 100000 habitantes. La literatura médica mundial refiere que una población con una incidencia que sobrepase los 20 por 100000 habitantes se considera una zona de alto riesgo (2).

En particular, la infección por Helicobacter pylori, los cambios en los mecanismos epigenéticos de regulación génica, la dieta y el consumo de alcohol y de tabaco se consideran los principales factores para el desarrollo de cáncer gástrico $(3,4)$. Para el estudio de esta patología, se ha establecido un modelo secuencial que explica la progresión de la carcinogénesis gástrica, en la que la mucosa normal sufre alteraciones como gastritis superficial, atrofia o pérdidas de glándulas, metaplasia intestinal (que, primero, se asemeja a la histología del intestino delgado, luego a la del grueso) y, finalmente, displasia, que, en conjunto, se conocen como lesiones precursoras de malignidad gástrica $(4,5)$. En la actualidad, a la dieta se le ha atribuido alrededor de una tercera parte de los casos de cáncer gástrico, razón por la cual cobra cada vez más importancia en el estudio de la etiopatogenia de las enfermedades crónicas, de ahí la importancia de la valoración del consumo de alimentos y el análisis del consumo de nutrientes para prever el estado de salud de una comunidad y orientar acciones de prevención (6).

El objetivo de este estudio fue determinar la asociación entre los hábitos alimentarios y la presencia de lesiones precursoras de malignidad gástrica en personas que acuden a consulta de gastroenterología en el Centro de Investigación de Enfermedades Digestivas de la ciudad de Pasto.

\section{METODOLOGÍA}

Se realizó un estudio analítico transversal, en el cual se analizaron variables sociales, demográficas, antropométricas y alimentarias, además del hábito de fumar, el consumo de alcohol, la incorporación de sal a las comidas servidas y variables histológicas para diagnosticar la presencia o ausencia de lesiones precursoras de malignidad gástrica.

Se encuestaron 231 pacientes remitidos por diferentes instituciones de salud para la realización de endoscopia en el Centro de Investigación de Enfermedades Digestivas de la ciudad de Pasto. Para la selección de la muestra, se utilizó el método de muestreo no probabilístico, bajo el criterio de accesibilidad, teniendo en cuenta la frecuencia de lesiones del 38,6\% reportada para Pasto en el año 2010 (2), con base en una población de 630 personas correspondientes al promedio de sujetos atendidos en este centro en los últimos 7 años, y con una precisión del 5\%. La muestra se calculó mediante la siguiente fórmula:

$$
\mathrm{n}=\frac{\mathrm{Z}_{1-\frac{\alpha}{2}}^{2} \cdot \mathrm{Npq}}{(\mathrm{N}-1) \mathrm{d}^{2}+\mathrm{Z}_{1-\frac{\alpha}{2}}^{2} p q}
$$

$\mathbf{n}=$ Tamaño muestral.

$\mathbf{N}$ = Tamaño de la población correspondiente al promedio de sujetos atendidos en el Centro de Investigación de Enfermedades Digestivas en los últimos 7 años.

$\mathbf{Z}=$ Valor correspondiente a la distribución de Gauss 1,96 para $\alpha=0,05$.

$\mathbf{p}=$ Frecuencia de lesiones precursoras de malignidad gástrica.

$\mathbf{q}=1-\mathrm{p}$.

d = Error máximo permisible (5\%).

Para la recolección de la información, se hizo uso de un cuestionario de frecuencia de consumo, con una lista cerrada de alimentos propios de la región, agrupados por su valor nutricional y no por su origen, de tal manera que los alimentos que pertenecen a cada grupo son intercambiables y mantienen constantes los valores de energía y macronutrientes, según principios estadísticos de variabilidad y homogeneidad; esto, siempre y cuando se siga las cantidades indicadas (porciones estandarizadas) para cada uno de ellos, lo que se conoce como sistema de intercambio de alimentos. A través de este sistema, se logró una estimación más certera sobre el número de intercambios consumidos de cada grupo de alimentos en un día. La recolección de los datos estuvo a cargo de los investigadores del proyecto y auxiliares de investigación, quienes recibieron capacitación y estandarización respecto de la aplicación del instrumento. La recolección se realizó antes de la endoscopia digestiva.

Para el análisis estadístico, se utilizó el programa Stata V.10.1 para Windows. Se realizó un análisis descriptivo $y$, para las variables de naturaleza cuantitativa, se calcularon medidas de tendencia central, dispersión o posición dependiendo de la distribución de los datos. Las variables de naturaleza cualitativa se resumieron con medidas de frecuencias. Se calculó la odds ratio (oportunidad relativa) cruda y ajustada mediante regresión logística binaria, con sus respectivos intervalos de confianza al 95\% (IC del 95\%). 
Las variables que se han reportado como posibles factores de riesgo o de protección se incluyeron en el modelo ajustado. Se consideraron significativos los valores $\mathrm{p}<0,05$.

Esta investigación es considerada sin riesgo según lo establecido en la Resolución 008430 de 1993 del Ministerio de Salud de Colombia. Las variables incluidas en la encuesta no modificaban ni trataban aspectos sensibles de la conducta. El estudio obtuvo aprobación por parte del Comité de Ética de Investigación en Seres Humanos de la Universidad CES (Acta n. ${ }^{\circ} 81$ del 28 de septiembre de 2015; código del proyecto: 481$)$.

\section{RESULTADOS}

El 35,5\% de los 231 pacientes encuestados fueron diagnosticados histológicamente con lesiones precursoras de malignidad gástrica; la edad promedio no superó los 46 años; predominó el sexo femenino con $57,1 \%$; el $32,5 \%$ tenía una formación de nivel profesional y el $58 \%$ estaba afiliado al régimen subsidiado. Estas lesiones se asociaron con el consumo de cereales y grasas por encima de los 10 y 8,5 intercambios (OR 2,20; IC del 95\%: 1,08-4,47 y OR 2,52; IC del 95\%: 1,28-4,94), respectivamente. Estos números de intercambios son los valores máximos de intercambios recomendados en las guías alimentarias para la población colombiana para estos grupos de alimentos, las cuales se tomaron como referencia. La incorporación de sal a las comidas servidas aumenta la probabilidad de presentar lesiones a nivel de la mucosa gástrica en 1,94 (IC del 95\%: 1,03-3,66). El consumo regular de vegetales por encima de los 2 intercambios reduce la probabilidad de presentar lesiones precursoras de malignidad (OR 0,53; IC del 95\%: $0,27-0,99)$. Aunque en el modelo crudo el consumo por encima de 2 intercambios de frutas se asoció con la disminución de estas lesiones (OR 0,55; IC del 95\%: 0,31-0,97), al hacer el ajuste, no se encontró asociación (OR 0,67; IC del 95\%: 0,36-1,26). Ninguno de los otros grupos de alimentos, el consumo de alcohol y cigarrillo ni el sexo mostraron asociación significativa con la presencia de lesiones precursoras de malignidad gástrica (Tabla 1).

\section{DISCUSIÓN}

Los estilos de vida, incluida la dieta, se reconocen como determinantes potencialmente importantes del aumento del riesgo de desarrollo de enfermedades crónicas como el cáncer. Se ha establecido que un 35\% de las muertes por esta enfermedad podrían evitarse al incorporar hábitos más saludables y controlar la obesidad (7). Los vínculos entre la dieta y el cáncer son complejos, y no se ha estudiado en profundidad su participación en las vías hacia la carcinogénesis (8). Se ha propuesto que el cáncer, antes de ser clínicamente evidente, tiene su asiento en etapas secuenciales bien definidas: gastritis atrófica multifocal (pérdida de glándulas gástricas) $\rightarrow$ metaplasia intestinal completa $\rightarrow$ metaplasia intestinal incompleta $\rightarrow$ displasia de bajo grado y, finalmente, cáncer invasivo. A estos estadios, que implican una predisposición cronológica, pero no definitiva, a la aparición del cáncer gástrico se los ha denominado lesiones precursoras de malignidad gástrica, cuya prevalencia encontrada para Pasto en el 2012 fue del 38,6\% (3, 4).

Los resultados de la presente investigación mostraron que las personas que consumen cereales y grasas por encima de lo recomendado en las guías alimentarias para Colombia tienen mayor probabilidad de presentar lesiones precursoras de malignidad gástrica, en comparación a quienes tienen un consumo ajustado a los valores diarios recomendados para estos dos grupos de alimentos. Se ha estimado que, aproximadamente, más del $60 \%$ de la composición química de cada 100 gramos de la porción comestible de distintos granos de cereales corresponde a hidratos de carbono; una dieta saludable y equilibrada tiene entre un $55 \%$ y un $66 \%$ de estas biomoléculas (9). Sin embargo, su consumo elevado parece estar asociado con alteraciones en el metabolismo de la glucosa, la hiperinsulinemia, la resistencia a la insulina, cambios en la regulación de factores de crecimiento insulínico (IGF tipo 1 y 2), modificación del metabolismo de las hormonas sexuales, inflamación crónica, modificaciones en la producción de adipoquinas y factores de crecimiento vascular por el tejido adiposo, estrés oxidativo y alteraciones en la función inmunitaria, que, en conjunto, pueden propiciar eventos celulares que desencadenen la génesis de un tipo de cáncer, como puede ser el gástrico $(9,10)$.

Recientemente, se ha comprobado que una dieta rica en hidratos de carbono puede contribuir hasta un $72 \%$ de aporte energético diario y estimular la síntesis de IGF-1 por el incremento inusitado en la concentración de la insulina en plasma. Este evento en particular puede ser el responsable de tener un efecto mutagénico y promotor de la tumurogénesis en cascada, de tal forma que la hiperinsulinemia puede ser el origen de la asociación existente entre las lesiones de la mucosa gástrica y el hábito de una alta ingesta energética (11). Un estudio realizado en el sur de la India determinó que el consumo frecuente y habitual de carbohidratos aumenta el riesgo de cáncer gástrico; el $44 \%$ de los casos presentaba un consumo alto, frente al $30 \%$ de quienes estaban en el grupo control (12).

Se ha determinado que la ingesta de cereales refinados, utilizados para la elaboración de pan blanco, pasta y arroz, influye significativamente en el aumento del riesgo de cáncer gástrico en una población del norte de Italia, pues se concluyó que existe una relación directa entre un alto índice glucémico y el riesgo de desarrollar este tipo de 
Tabla 1. Factores asociados con la presencia de lesiones precursoras de malignidad gástrica.

\begin{tabular}{|c|c|c|c|c|c|c|c|c|c|c|c|c|}
\hline \multirow[t]{3}{*}{ Variable } & \multicolumn{4}{|c|}{$\begin{array}{l}\text { Lesiones precursoras de } \\
\text { malignidad gástrica }\end{array}$} & \multirow{2}{*}{\multicolumn{2}{|c|}{ otal }} & \multicolumn{3}{|c|}{ Modelo crudo } & \multicolumn{3}{|c|}{ Modelo ajustado } \\
\hline & \multicolumn{2}{|c|}{ Sí } & \multicolumn{2}{|c|}{ No } & & & \multirow[b]{2}{*}{$\begin{array}{l}\text { Odds ratio } \\
\text { (OR) }\end{array}$} & \multirow[b]{2}{*}{$\begin{array}{l}\text { IC del } \\
95 \%\end{array}$} & \multirow[b]{2}{*}{$\begin{array}{c}\text { Valor } \\
p\end{array}$} & \multirow[b]{2}{*}{$\begin{array}{l}\text { Odds ratio } \\
(\mathrm{OR})^{*}\end{array}$} & \multirow[b]{2}{*}{$\begin{array}{l}\text { IC del } \\
95 \%\end{array}$} & \multirow[b]{2}{*}{$\begin{array}{c}\text { Valor } \\
p\end{array}$} \\
\hline & $\mathrm{n}$ & $\%$ & $\mathrm{n}$ & $\%$ & $\mathrm{n}$ & $\%$ & & & & & & \\
\hline \multicolumn{13}{|l|}{ Sexo } \\
\hline Hombre & 37 & 45,1 & 62 & 41,6 & 99 & 42,9 & 1,15 & $0,67-1,99$ & 0,606 & 0,93 & $0,47-1,81$ & 0,827 \\
\hline Mujer & 45 & 54,9 & 87 & 58,4 & 132 & 57,1 & 1,00 & & & & & \\
\hline \multicolumn{13}{|l|}{ Cereales } \\
\hline Mayor a 10 intercambios & 64 & 78,0 & 88 & 59,1 & 152 & 65,8 & 2,45 & $1,33-4,56$ & 0,004 & 2,20 & $1,08-4,47$ & 0,029 \\
\hline 6-10 intercambios & 18 & 22,0 & 61 & 40,9 & 79 & 34,2 & 1,00 & & & & & \\
\hline \multicolumn{13}{|l|}{ Verduras } \\
\hline Mayor de 2 intercambios & 21 & 25,6 & 62 & 41,6 & 83 & 34,6 & 0,43 & $0,28-0,87$ & 0,015 & 0,53 & $0,27-0,99$ & 0,049 \\
\hline $0-2$ intercambios & 61 & 74,4 & 87 & 58,4 & 148 & 65,4 & 1,00 & & & & & \\
\hline \multicolumn{13}{|l|}{ Frutas } \\
\hline Mayor de 2 intercambios & 25 & 30,5 & 66 & 44,3 & 91 & 39,4 & 0,55 & $0,31-0,97$ & 0,040 & 0,67 & $0,36-1,26$ & 0,210 \\
\hline $0-2$ intercambios & 57 & 69,5 & 83 & 55,7 & 140 & 60,6 & 1,00 & & & & & \\
\hline \multicolumn{13}{|l|}{ Leguminosas } \\
\hline Mayor de 2 intercambios & 21 & 25,6 & 36 & 24,2 & 57 & 24,7 & 1,08 & $0,58-2,01$ & 0,807 & 0,70 & $0,34-1,42$ & 0,319 \\
\hline $0-2$ intercambios & 61 & 74,4 & 113 & 75,8 & 174 & 75,3 & 1,00 & & & & & \\
\hline \multicolumn{13}{|l|}{ Lácteos } \\
\hline Mayor de 2 intercambios & 35 & 42,7 & 62 & 41,6 & 97 & 42,0 & 1,04 & $0,61-1,80$ & 0,874 & 0,86 & $0,46-1,61$ & 0,632 \\
\hline $0-2$ intercambios & 47 & 57,3 & 87 & 58,4 & 134 & 58,0 & 1,00 & & & & & \\
\hline \multicolumn{13}{|l|}{ Grasas } \\
\hline Mayor de 8,5 intercambios & 47 & 57,3 & 51 & 34,9 & 98 & 42,4 & 2,57 & $1,48-4,49$ & 0,001 & 2,52 & $1,28-4,94$ & 0,007 \\
\hline 6-8,5 intercambios & 35 & 42,7 & 98 & 65,1 & 133 & 57,6 & 1,00 & & & & & \\
\hline \multicolumn{13}{|l|}{ Carnes } \\
\hline Mayor de 2 intercambios & 64 & 78,0 & 116 & 77,9 & 180 & 79,9 & 1,01 & $0,53-1,94$ & 0,973 & 0,86 & $0,40-1,86$ & 0,708 \\
\hline $0-2$ intercambios & 18 & 22,0 & 33 & 22,1 & 51 & 22,1 & 1,00 & & & & & \\
\hline \multicolumn{13}{|l|}{ Azúcares } \\
\hline Mayor de 5 intercambios & 59 & 72,0 & 93 & 62,4 & 152 & 65,8 & 1,54 & $0,86-2,77$ & 0,144 & 0,77 & $0,37-1,58$ & 0,472 \\
\hline $0-5$ intercambios & 23 & 28,0 & 56 & 37,6 & 79 & 34,2 & 1,00 & & & & & \\
\hline \multicolumn{13}{|l|}{ Alcohol } \\
\hline Sí consume & 22 & 26,8 & 33 & 22,1 & 55 & 23,8 & 1,29 & $0,69-2,40$ & 0,424 & 0,70 & $0,33-1,49$ & 0,358 \\
\hline No consume & 60 & 73,2 & 116 & 77,9 & 176 & 76,2 & 1,00 & & & & & \\
\hline Cigarrillo & & & & & & & & & & & & \\
\hline Sí consume & 20 & 24,4 & 22 & 14,8 & 42 & 18,2 & 1,86 & $0,94-3,66$ & 0,069 & 1,27 & $0,55-2,96$ & 0,577 \\
\hline No consume & 62 & 75,6 & 127 & 85,2 & 189 & 81,8 & 1,00 & & & & & \\
\hline Sal & & & & & & & & & & & & \\
\hline Sí incorpora & 47 & 57,3 & 55 & 36,9 & 102 & 44,2 & 2,29 & $1,32-3,98$ & 0,003 & 1,94 & 1,03-3,66 & 0,040 \\
\hline No incorpora & 35 & 42,7 & 94 & 63,1 & 129 & 55,8 & 1,00 & & & 1,00 & & \\
\hline
\end{tabular}

*Regresión logística binaria; se generó un modelo ajustado con todas las variables presentadas.

cáncer (OR 2,5; IC del 95\%: 1,3-4,9) (13). La explicación que prevalece en estas dos investigaciones sugiere que estos alimentos promueven la producción de insulina y la acti- vación del IGF-1, se estimula la proliferación, los factores de crecimiento celular y se inhibe la apoptosis al promover la supervivencia celular tumoral $(12,13)$. Otro estudio, 
realizado en Colombia en el departamento de Nariño, que buscaba explorar la asociación entre la concentración de selenio y el cáncer gástrico, estableció que el consumo de papas cocidas entre 6 y 7 veces por semana es un factor de riesgo desencadenante de cáncer gástrico en hombres (14). Asimismo, otras investigaciones han encontrado que habas, cubios, nabos y papas, alimentos muy comunes y de fácil adquisición en la zona andina del país, contienen gran cantidad de almidones complejos que resultan abrasivos por su difícil digestión y pueden generar posiblemente irritaciones en la mucosa gástrica y, en forma subsiguiente, lesiones precursoras de malignidad (15). Martínez y colaboradores reportaron que una dieta de "pan y huevo" se asoció positivamente con gastritis crónica atrófica (OR 2,69; IC del 95\%: 1,2-6,08) y metaplasia (OR 4,15; IC del $95 \%: 1,79-9,66)(16)$.

Por otro lado, se ha encontrado que existe relación entre el riesgo de cáncer y el total de grasa y ácidos grasos saturados de la dieta. En esta investigación, se encontró que quienes consumen grasas por encima de los 8,5 intercambios tienen mayor probabilidad de presentar lesiones gástricas, en comparación con quienes lo hacen en un rango adecuado según las recomendaciones de las guías alimentarias. Esto puede deberse, posiblemente, a que los lípidos afectan directamente algunas funciones celulares, entre ellas, la fluidez u homeostasis de la membrana celular, el metabolismo de las prostaglandinas y la síntesis de radicales peróxido. Una dieta en la que predominen las grasas puede producir cambios en los receptores hormonales, un crecimiento celular anormal y modificaciones a nivel intracelular; además, se ha reportado que su consumo frecuente puede provocar cambios en la composición de la bilis e iniciar la activación de componentes de la dieta a sustancias con capacidad carcinogénica $(17,18)$.

En las islas Zhoushan (China), se llevó a cabo una investigación que determinó que el consumo elevado de grasas saturadas, especialmente entre hombres, aumenta el riesgo de cáncer gástrico (OR 3,24; IC del 95\%: 1,11-9,49) (19). De igual manera, se identificó una asociación positiva con la ingesta total de grasas (OR 1,33; IC del 95\%: 1,12-1,57) y el riesgo de cáncer gástrico (20). Otro de los reportes más recientes, que buscó determinar una asociación entre la ingesta de macronutrientes y el riesgo de cáncer gástrico en una población de América del Norte, encontró que el consumo de grasa total, grasa saturada y colesterol se asoció significativamente con el riesgo de cáncer gástrico, con OR calculadas de 1,58 (IC del 95\%: 1,13-2,20); 1,86 (IC del 95\%: 1,37-2,52) y 1,75 (IC del 95\%: 1,36-2,25), respectivamente (21).

Actualmente, la investigación se ha centrado en determinar las interacciones entre los factores dietéticos y el desarrollo de cáncer gástrico, y se ha identificado la posible exis- tencia de cierta relación entre la alimentación, la etiología de este tipo de cáncer y su prevención. La conclusión que prevalece en la mayoría de los estudios es que una dieta rica en frutas y verduras reduce el riesgo de desarrollar cáncer gástrico (22). Riboli y Norat encontraron que por cada 100 $\mathrm{g}$ de aumento en la ingesta de vegetales, la estimación del riesgo relativo fue de 0,81 (IC del 95\%: 0,75-0,87) y, para el consumo de frutas, fue de 0,74 (IC del 95\%: 0,69-0,81), lo que indica un efecto protector (23). Otra investigación concluyó que el consumo de frutas y verduras demostró asociaciones inversas con la aparición de cáncer gástrico (OR 0,72; IC del 95\%: 0,65-0,80 y OR 0,75; IC del 95\%: $0,60-0,95$, respectivamente) (20).

En un estudio sobre dieta y cáncer gástrico, realizado en el año 2007 en la Facultad de Medicina de la Universidad Nacional de Cheju (Corea), se analizaron seis estudios hospitalarios de casos y controles, seis estudios de casos y controles basados en la comunidad y dos estudios de cohortes, y se demostró una reducción del $28 \%$ en el riesgo de cáncer gástrico asociado con un alto consumo de frutas cítricas (OR 0,72; IC del 95\%: 0,64-0,81) (24). Para el presente estudio, se determinó que la razón de prevalencia ajustada para el consumo de verduras fue de 0,67 (IC del 95\%: 0,450,99 ) y de 0,77 (IC del 95\%: 0,53-1,13) para el consumo de frutas. Sin embargo, se encontró que solo el consumo regular de verduras por encima de los 2 intercambios se asoció con la disminución en el riesgo de prevalencia de presentar lesiones precursoras de malignidad gástrica.

Según nuestros resultados, se puede establecer que el consumo de leguminosas, carnes, lácteos y azucares no se asoció de forma significativa con la presencia de lesiones precursoras de malignidad; sin embargo, un estudio que buscó determinar la asociación entre la dieta y la carcinogénesis gástrica encontró que consumir carnes procesadas se asoció positivamente con gastritis crónica atrófica multifocal (OR 3,61; IC del 95\%: 1,46-8,92) y cáncer gástrico (OR 3,10; IC del 95\%: 0,97-9,8), a diferencia de las leguminosas, que tuvieron una relación inversa con el cáncer gástrico (OR 0,25; IC del 95\%: 0,08-0,81).

Para el consumo de sal, especialmente la incorporación de sal a las comidas servidas, se encontró que quienes utilizan el salero tienen una prevalencia de riesgo estadísticamente significativo de desarrollo de lesiones precursoras de malignidad gástrica. Se evaluó el consumo de sal en diferentes formas, dentro de las que se incluyen el consumo de alimentos salados y la incorporación de sal a las comidas después de su cocción, así como mediante pruebas bioquímicas para medir la excreción de sodio en la orina. Según un grupo de expertos del Fondo Mundial de Investigación del Cáncer (WCRF, por sus siglas en inglés), existe consenso en determinar la relación del consumo de sal (depen- 
diente de la dosis) y la incidencia de cáncer gástrico. Se ha reportado que un aumento de alrededor del $8 \%$ en el consumo total de sal (g/día) aumenta el riesgo de desarrollar lesiones gástricas (25).

Para quienes tienen el hábito de consumir cigarrillo y alcohol, en esta investigación no se encontraron diferencias estadísticas que asocien su consumo con la presencia de lesiones gástricas. No obstante, el tabaquismo es una causa establecida de cáncer gástrico, pero parece actuar como un factor de riesgo moderado, en comparación con otros cánceres asociados con el consumo de tabaco. Un metaanálisis realizado por Ladeiras y colaboradores, que incluyó solo estudios prospectivos, mostró una estimación del riesgo de cáncer gástrico de 1,62 (IC del 95\%: 1,50-1,75) y 1,20 (IC del 95\%: 1,01-1,43) para hombres y mujeres fumadores, respectivamente (26).

La mayoría de los estudios epidemiológicos indican una relación entre determinados hábitos alimentarios y el desarrollo del cáncer gástrico. Algunos hábitos aumentan el riesgo de desarrollarlo, y otros ofrecen un efecto protector; sin embargo, los estudios sobre alimentación y lesiones precursoras de malignidad gástrica siguen siendo escasos. Este estudio, por lo tanto, está orientado a brindar una información más cercana del comportamiento de los hábitos alimentarios en relación con los estadios previos a la aparición del cáncer gástrico, detectados mediante estudios histopatológicos. En cuanto al instrumento utilizado para la recolección de la información (cuestionario de frecuencia de consumo), este no permite estimar una cantidad exacta de alimentos o nutrientes, y la información suministrada depende de la memoria de los encuestados. El instrumento no se validó para la población del departamento de Nariño, pero el listado de alimentos se ajustó de acuerdo con los hábitos alimentarios de la región. Sin embargo, la ventaja del instrumento seleccionado radica en que puede obtenerse información sobre la alimentación habitual, y se reconoce la utilidad del cuestionario de frecuencia de consumo de alimentos en estudios epidemiológicos que relacionan la dieta con la enfermedad o con factores de riesgo.

La muestra seleccionada se obtuvo de pacientes que, por presentar sintomatología gástrica, acudieron a consulta especializada para la realización de endoscopia digestiva. Por lo tanto, la prevalencia de lesiones precursoras de malignidad posiblemente estaría sobrestimada respecto de la población general. El sesgo de información se controló al aplicar el cuestionario antes de realizar la endoscopia digestiva y al tener el resultado definitivo de la presencia o ausencia de lesiones precursoras de malignidad a través del estudio histopatológico. Por otra parte, las pérdidas de información fueron mínimas.

\section{REFERENCIAS}

1. Torre LA, Bray F, Siegel RL, et al. Global cancer statistics, 2012. CA: Cancer Journal for Clinicians. 2015;65(2):87108. Doi: https://doi.org/10.3322/caac.21262

2. Bedoya UA, Sansón GF, Yépez Fuertes VY, et al. Prevalencia y severidad de las lesiones precursoras de malignidad en una área de alto riesgo de cáncer gástrico. Pasto 2012. Revista Colombiana de Gastroenterologia. 2012;27:275-81.

3. González CA, Agudo A. Carcinogenesis, prevention and early detection of gastric cancer: where we are and where we should go. Int J Cancer. 2012;130(4):745-53. Doi: https:// doi.org/10.1002/ijc.26430

4. Piazuelo MB, Epplein M, Correa P. Gastric cancer: an infectious disease. Infect Dis Clin North Am. 2010;24(4):85369. Doi: https://doi.org/10.1016/j.idc.2010.07.010

5. Correa P. Human gastric carcinogenesis: a multistep and multifactorial process. First American Cancer Society Award Lecture on Cancer Epidemiology and Prevention. Cancer Res. 1992;52(24):6735-40.

6. Anand P, Kunnumakara AB, Sundaram C, et al. Cancer is a preventable disease that requires major lifestyle changes. Pharm Res. 2008;25(9):2097-116. Doi: https://doi. org/10.1007/s11095-008-9661-9

7. Doll R, Peto R. The causes of cancer: quantitative estimates of avoidable risks of cancer in the United States today. J Natl Cancer Inst. 1981;66(6):1191-308. Doi: https://doi. org/10.1093/jnci/66.6.1192

8. Who J, Consultation FE. Diet, nutrition and the prevention of chronic diseases. World Health Organ Tech Rep Ser. 2003;916(i-viii).

9. Pérez-Guisado J. Hidratos de carbono, metabolismo de la glucosa y cáncer. Endocrinología y Nutrición. 2006;53(4):2525. Doi: https://doi.org/10.1016/S1575-0922(06)71099-3

10. Florini JR, Ewton DZ, Magri KA. Hormones, growth factors, and myogenic differentiation. Annu Rev Physiol. 1991;53:201-16. Doi: https://doi.org/10.1146/annurev. ph.53.030191.001221

11. Giovannucci E. Insulin, insulin-like growth factors and colon cancer: a review of the evidence. J Nutr. 2001;131(11):3109S-20S.

12. Laroiya I, Pankaja SS, Mittal S, et al. A study of Helicobacter pylori infection, dietary pattern and habits in patients with gastric cancer in South India. Asian Pacific Journal of Tropical Disease. 2012;2(1):24-6. Doi: https://doi. org/10.1016/S2222-1808(12)60006-1

13. Bertuccio P, Praud D, Chatenoud L, et al. Dietary glycemic load and gastric cancer risk in Italy. $\mathrm{Br} \mathrm{J}$ Cancer. 2009;100(3):558-61. Doi: https://doi.org/10.1038/ sj.bjc. 6604894

14. Camargo MC, Burk RF, Bravo LE, et al. Plasma selenium measurements in subjects from areas with contrasting gastric cancer risks in Colombia. Archives of Medical Research. 2008;39(4):443-51. Doi: https://doi.org/10.1016/j.arcmed.2007.12.004 
15. Rodríguez A, Alvarado J, Sandler R, et al. Asociación entre infección por Helicobacter pylori y cáncer gástrico en Colombia. Acta Med Col. 2000;25:112-6.

16. Martínez T, Hernández G, Rojas C. La dieta y su asociación con lesiones preneoplásicas y cáncer gástrico en una zona de alto riesgo para cáncer gástrico en Colombia I, 2000-2006. Rev Colomb Cancerol. 2008;12:74-88.

17. van den Brandt PA, Goldbohm RA. Nutrition in the prevention of gastrointestinal cancer. Best Pract Res Clin Gastroenterol. 2006;20(3):589-603. Doi: https://doi. org/10.1016/j.bpg.2006.04.001

18. Pierart C, Rozowsky J. Papel de la nutrición en la prevención del cáncer gastrointestinal. Revista Chilena de Nutrición. 2006;33(1):8-13. Doi: https://doi.org/10.4067/S071775182006000100001

19. Qiu JL, Chen K, Zheng JN, et al. Nutritional factors and gastric cancer in Zhoushan Islands, China. World J Gastroenterol. 2005;11(28):4311-6. Doi: https://doi. org/10.3748/wjg.v11.i28.4311

20. Pakseresht M, Forman D, Malekzadeh R, et al. Dietary habits and gastric cancer risk in north-west Iran. Cancer Causes Control. 2011;22(5):725-36. Doi: https://doi. org/10.1007/s10552-011-9744-5
21. Hu J, La Vecchia C, Negri E, et al. Macronutrient intake and stomach cancer. Cancer Causes Control. 2015;26(6):83947. Doi: https://doi.org/10.1007/s10552-015-0557-9

22. Nutritional aspects of the development of cancer. Report of the Working Group on Diet and Cancer of the Committee on Medical Aspects of Food and Nutrition Policy. Rep Health Soc Subj. 1998;48:1-274.

23. Riboli E, Norat T. Epidemiologic evidence of the protective effect of fruit and vegetables on cancer risk. Am J Clin Nutr. 2003;78(3):559S-69S.

24. Bae JM, Lee EJ, Guyatt G. Citrus fruit intake and stomach cancer risk: a quantitative systematic review. Gastric Cancer. 2008;11(1):23-32. Doi: https://doi.org/10.1007/s10120007-0447-2

25. De MartelC, FerlayJ, FranceschiS, etal. Globalburden of cancers attributable to infections in 2008: a review and synthetic analysis. The Lancet Oncology. 2012;13(6):607-15. Doi: https://doi.org/10.1016/S1470-2045(12)70137-7

26. Ladeiras-Lopes R, Pereira AK, Nogueira A, et al. Smoking and gastric cancer: systematic review and meta-analysis of cohort studies. Cancer Causes Control. 2008;19(7):689701. Doi: https://doi.org/10.1007/s10552-008-9132-y 